\title{
Topography of Si epitaxial monolayers obtained on Si (001) substrate by computer simulations
}

\author{
L. Pyziak ${ }^{1}$, W. Obermayr ${ }^{2}$, K. Zembrowska ${ }^{1}$, M. Kuzma ${ }^{1}$ \\ ${ }^{1}$ Institute of Physics, University of Rzeszow, 35-310 Rzeszow, Rejtana 16a, Poland \\ ${ }^{2}$ Technikum Joanneum Gmbh, Alte Poststrasse 149, A-8020 Graz, Austria \\ Phone: +48(17) 862 5628; fax: +48(17) 852 26792; e-mail: lpyziak@univ.rzeszow.pl
}

\begin{abstract}
Fractal analysis was used for the description of the geometry of the clusters formed within the Monte Carlo simulation of the first monolayer growth on Si substrate. Pulse laser deposition method was assumed for the epitaxy. Layers were obtained for various substrate temperatures varying in the range from $600 \mathrm{~K}$ to $800 \mathrm{~K}$. The topography of plane clusters formed were characterised by their fractal box-like dimension. The relation between this dimension and the shape of the clusters was addressed.
\end{abstract}

Keywords: silicon, epitaxial monolayer, Monte Carlo simulation, fractal characterisation.

Paper received 28.01.03; accepted for publication 16.06.03.

\section{Introduction}

Pulsed laser deposition method (PLD) of thin solid films fabrication [1,2] is very promising in various new material technologies such as electronics [3-6], metallurgy $[7,8]$, biology or even in medicine $[9,10]$, etc. The advantage of the method is a good control of the layer growing process by adjusting of wide set of technology parameters such as: the energy of laser beam, the substrate temperature, the pressure of ambient gas, etc. Thus the method is widely used for layer deposition not only of classical materials (metals, semiconductors) but also such specific compounds like high-temperature superconductors [11-13] new metal alloys [14-17], biocompatible coatings [18,19], laser crystals [20,21] and polymers [22]. The determination of the technology parameters of layer growth is realised either by the theoretical estimation or by in-situ monitoring during the layer deposition by RHEED [23]. An alternate method for this purpose is a numerical simulation of the layer growth presuming a set of parameter values [24-26]. By carrying out the simulations, we can establish the best conditions for epitaxial growth of layers.
The Monte Carlo method [28] is a very convenient tool for such computer simulations [29-31]. The model of the layer formation includes the following problems of the deposition process: structure of an incident flux (e.g. energy of atoms, space distribution), absorption and desorption, surface migration, etc. The growth characteristics such as particles nucleation type, the mode of layer growth, the shape of the clusters formed, the structure quality of layers should be investigated on each stage of layer formation. In this aim, we propose to apply the fractal characterisation of clusters formed in the initial stage of layer growth. The geometrical shape of non-regular planar objects is described by fractal dimension very well. By inspection of this parameter we can determine conditions of layer deposition, which are most favourable to epitaxial growth. The method will be applied to the investigation of $\mathrm{Si}$ clusters obtained in computer simulations of the layer formation by PLD on Si substrate in initial stage of growing.

The particular aim of our investigation was to study the effects of surface temperature on size and topography of clusters forming the first monolayer. 


\section{Pyziak et al.: Topography of Si epitaxial monolayers ...}

\section{Procedure of film deposition on Si (001) surface}

\author{
a. Structure and properties of (001) Si \\ substrate
}

Silicon crystallises in a diamond structure. Atoms of the (001) plane form a square lattice having lattice constant $d=a^{\sqrt{2}} / 2$, where $a$ is the lattice constant of the diamond structure. A distance $d$ is a distance between a second near-neighbours in a diamond structure. The structure of silicon crystals in the [001] direction is layered by (001) planes (Fig 1). An atom inside a crystal is surrounded by four other atoms, which are covalence bonded with it. Thus each atom placed on (001) surface is bonded with two atoms in the layer below (atoms designated by "1"). Such atoms may have no more than eight second nearest neighbours (atoms designated by "2") from which four are in the second nearest layer below and four are placed in the same surface layer. But with these last atoms it does not form any bounds. Nevertheless, two second - nearest neighbouring atoms on the surface of the crystal can bind together forming dimer [32]. These dimers may be broke up by atoms landed on the surface.

\section{b. Monte Carlo simulation of the reconstruction of (001) Si surface}

In the thin layer growing process from vapour phase or particularly in a pulse laser deposition method (PLD) the atoms falling on a substrate have a kinetic energy $E_{k}$ obtained in an evaporation or ablation processes. Then these atoms diffuse on a substrate surface. The probability $p$ of the jumping of an atom from one lattice site to the other one is given by [32-34]:

$$
p=A \exp \left\{-\frac{E_{a}}{k_{B} T}\right\}
$$

where $A$ is a hopping rate and it was assumed to be $10^{13} \mathrm{~s}^{-1}$, $E_{a}$ is a surface diffusion energy. Surface diffusion energy

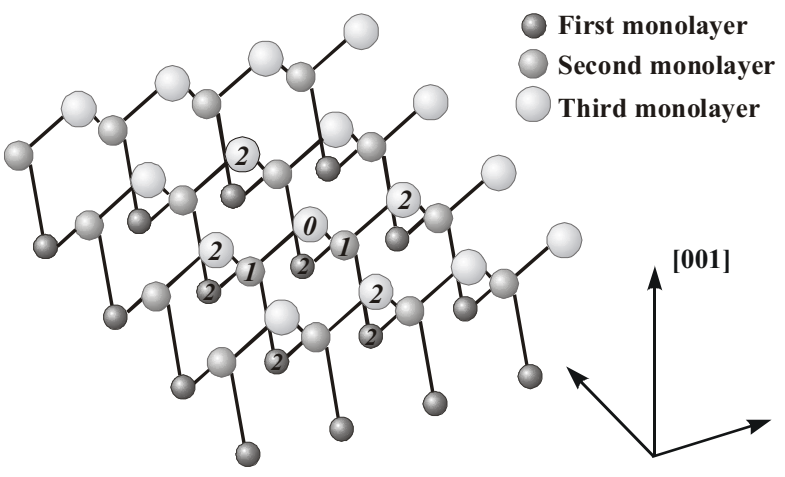

Fig. 1. Structure of silicon crystal in the [001] direction. consists of two terms given by interaction energies $E_{1}$ and $E_{2}$ of the adatom with nearest-neighbour and with second nearest-neighbour atoms respectively:

$E_{a}=n_{1} E_{1}+n_{2} E_{2}$

where $n_{1}$ and $n_{2}$ is the numbers of corresponding neighbours.

In the formulae (1) $T$ is the temperature of the substrate, a very important parameter of the PLD method.

In the Monte Carlo simulation procedure of layer growth [27-31] the square table consisting of $\mathrm{L} \times \mathrm{L}$ sites was assumed to be a representative of a crystal surface lattice of a Si substrate. The values 0 or 1 attached to each site correspond to the empty or occupied site by an adatom. The surface migration of an adatom on a (001) plane of the Si lattice was obtained via the proper transition rules imposed on the site-to-site hopping probability of adatoms [31]. We have assumed that atoms can jump only to the nearest sites on the same layers. For the square lattice we have therefore four directions for the hopping. However, an atom cannot jump to a site which is already occupied by another atom. On the hopping possibilities the two-dimensional Born-Karmann conditions was imposed. This means that the atom from the last column (or row) can hop to the first one in the direction of jumping to the right (to the down). Similarly this is also valid for the first column and row.

The simulation procedure consists of two main parts. The first step is a random deposition of a number $N$ of atoms coming from an atom beam on the empty flat surface of a substrate. This step is realised by a random generation of the $(x, y)$ site coordinates and denoting this site by a number 1 , which means that the adatom is just placed there. The generation is repeated $\mathrm{N}$-times.

In the next step one atom from the set of atoms deposited on the surface is chosen randomly for moving. For this atom the probability of jumping is calculated according to the formulae (1). This probability depends only on the site, which is occupied by this atom (depends on the number $n_{1}$ and $n_{2}$ of nearest- and second nearestneighbour atoms respectively), but it does not depend on the site to which the atom will jump. The calculated value $\mathrm{p}$ is compared with a number $P$ chosen randomly from the range $[0,1]$. The atom is qualified for hopping if $p \geq$ $P$. In the opposite case this atom is not moved and the next particle is randomly drawn for which the procedure is repeated. If the atom is qualified for moving, the direction of jumping is randomly chosen from these, which are allowed for this site. The probability of jumping to the each allowed direction is the same. After moving this particle to the new place the next particle is randomly chosen and the second step is repeated.

\section{Fractal dimension of nonregular flat struc- tures}

The mathematical characterisation of the shape of irregular objects is done by their fractal dimension. In general 


\section{Pyziak et al.: Topography of Si epitaxial monolayers ...}

it is the Hausdorff dimension. In the particular case of quasi-homogeneous flat structures it is a box-like dimension $D_{b}$ which is one from the Mandelbrot fractal dimensions [36-38]. In order to determine this dimension we place the structure (cluster) studied on a regular two-dimensional square lattice with lattice constant $s$ and we count the "boxes" of the lattice, which cover cluster in even the smallest part. That way we receive the $N(s)$ number of boxes. Next, we gradually diminish the size of the lattice constant $s$ and we determine the corresponding numbers $N(s)$. For convenience the series of lattices are chosen such that the sizes of boxes in successive lattices decrease by a factor two. Results of measurements show the exponential law:

$$
N(s)=s^{D_{b}}
$$

By present results received in a logarithmic coordinate system and approximating them by a linear function we determine the box-like dimension $D_{b}$ as the tangent of the slope of the line.

On a surface, the box-like dimension $D_{b}$ does never excess the value 2, which is the Euclidean dimension for two-dimensionality. It is also worth mentioning that for tightly packed structures (smooth shoreline, lack of holes) the box-like dimension is close to this value 2. Contrary, for non-compact morphologies of flat clusters (rough edges, highly anisotropic shapes, great number of lakes) this dimension is less then value 2 and not much greater then value 1 .
Calculations were performed by computer program. The program allows to determine the size of the clusters (the number of atoms in cluster), to calculate fractal dimension of cluster, and the average fractal dimension of clusters belonging to one of the three sets of clusters with respect to size as described bellow.

\section{Results and discussion}

The results of simulations were obtained for the following parameters: $L=50, N=1000$ or 1300 . Energies $E_{1}$ and $E_{2}$ were taken as $0.6 \mathrm{eV}$ and $0.1 \mathrm{eV}$ respectively [25]. Substrate temperature for different cases was changed by $10 \mathrm{~K}$ in the range $600-800 \mathrm{~K}$. The layers were deposited by one laser pulse ablating $N=1000$ or 1300 particles and these values were estimated from typical experimental rate of layer growth [1]. The average kinetic energy of an atom on a substrate surface $E_{k}=1,5 \mathrm{eV}$ was estimated from the thermal sputtering $[39,40]$ processed by YAG: $\mathrm{Nd}^{3+}$ laser (power density of laser beam $106 \mathrm{~W} / \mathrm{cm}^{2}$, pulse duration $40 \mathrm{~ns}$, the diameter of laser beam spot $3 \mathrm{~mm}$ ). The layers obtained consist of one-monolayer flat clusters. Fig. 1 presents examples of layers obtained for $N=1000$ (1300) and for substrate temperatures $600 \mathrm{~K}$, $730 \mathrm{~K}$ and $800 \mathrm{~K}$.

For each substrate temperature the size of clusters, the number of clusters $M$ belongs to one of the following three size ranges: small (20-50 atoms), medium (51-100
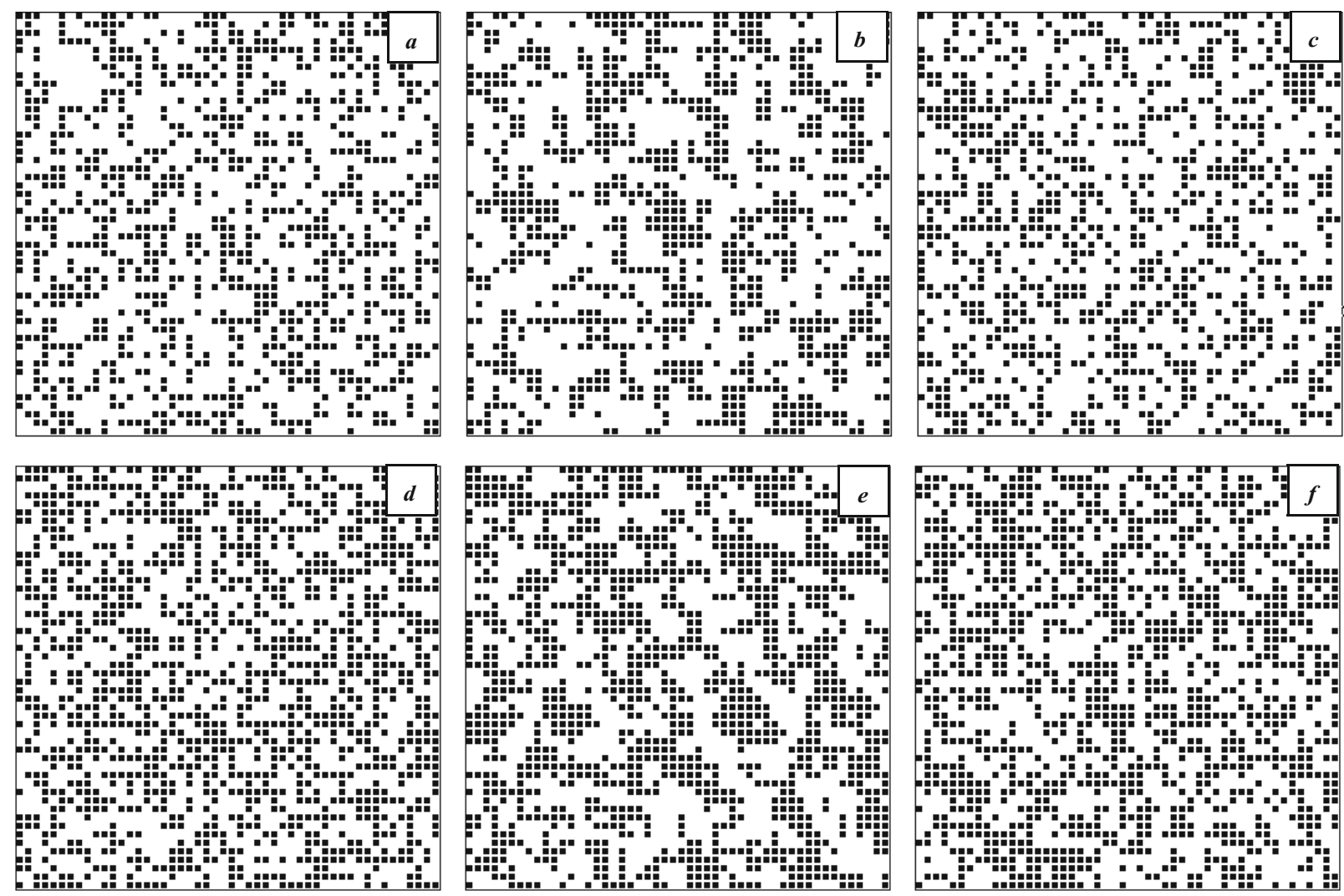

Fig. 2. Silicon layers obtained on (001) Si surface in various substrate temperatures: $a-600 \mathrm{~K}, b-730 \mathrm{~K}, c-800 \mathrm{~K}$ for $N=1000$ atoms and $d-600 \mathrm{~K}, e-730 \mathrm{~K}, f-800 \mathrm{~K}$ for $N=1300$ atoms. 


\section{Pyziak et al.: Topography of Si epitaxial monolayers ...}

atoms) or large (more than 100 atoms), were determined. Very small clusters consisting of less than 20 atoms were not taken into account. The results are presented in Fig. 3.

One can see that in the entire range of temperature considered there are very small clusters (20-50), and their maximum number is at about $650 \mathrm{~K}$ in both cases $N=1000$ and $N=1300$. With increasing substrate temperature this number decreases, because more medium and large clusters appear. In the temperature range 730 $750 \mathrm{~K}$ we have maximal number of large clusters. For temperatures above $780 \mathrm{~K}$ there are formed mainly small clusters again like in low temperatures. In the case $N=1300$ (Fig. 3 b) the temperature region about $750 \mathrm{~K}$ is very promising also due to the evidence decreasing of number of small as well as middle cluster, what produce nice layer consisting mainly large clusters. From these results we find, that the best temperature range ("temperature window") for our epitaxy is the range 730-750 K, since at these temperatures the largest clusters are formed.

Another important influence on the quality of the layer is given by the geometry of clusters. Therefore the fractal inspection for each cluster as well as for each size group of clusters was carried out. In Fig. 4 we present some examples of measurements of fractal dimension for clusters obtained at temperatures of $600 \mathrm{~K}, 610 \mathrm{~K}, 620 \mathrm{~K}(a) ; 680 \mathrm{~K}$ to $710 \mathrm{~K}(b) ; 730 \mathrm{~K}, 740 \mathrm{~K}(c)$ and $760 \mathrm{~K}, 770 \mathrm{~K}(d)$. One can see that generally the fractal dimension is small about 1,2 for all clusters formed at a given substrate temperature. This dimension increases considerably for temperatures in the range of $730-760 \mathrm{~K}$ and for large clusters (up to $\left.D_{b} \approx 1,5\right)$.
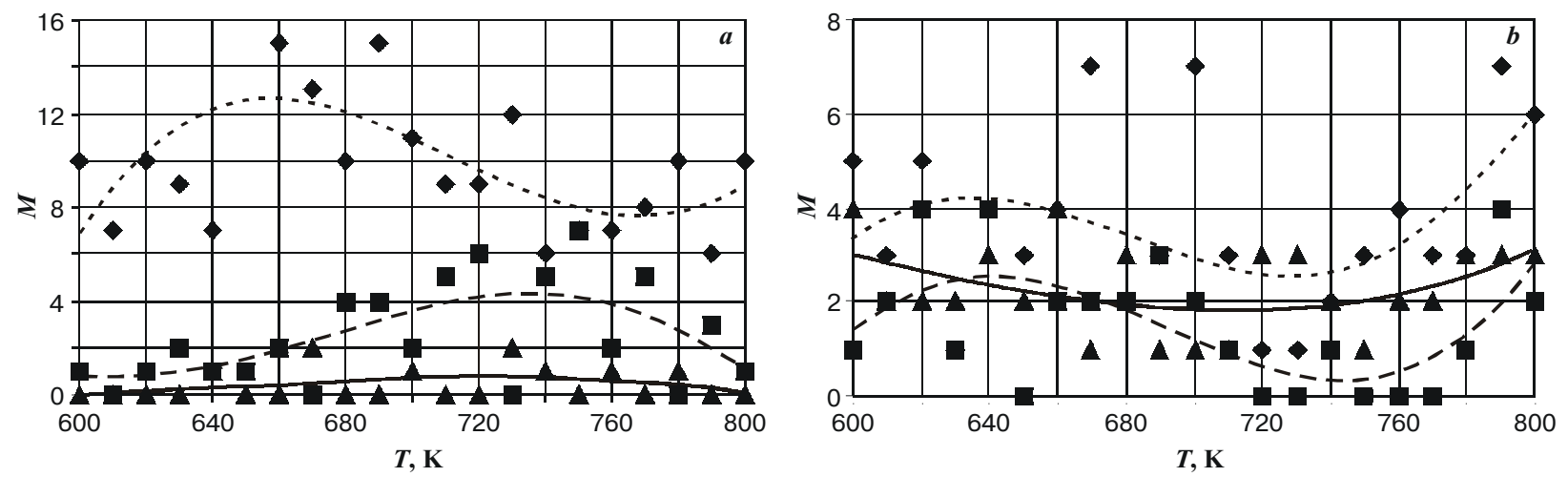

Fig. 3. Dependence of number of clusters (having size: small: 20-50 atoms “", middle: $51-100$ atoms "ஐ”, large: more then 100 atoms " $\boldsymbol{\Delta} ")$ on the substrate temperature for two simulation procedure $N=1000(a), N=1300(b)$.
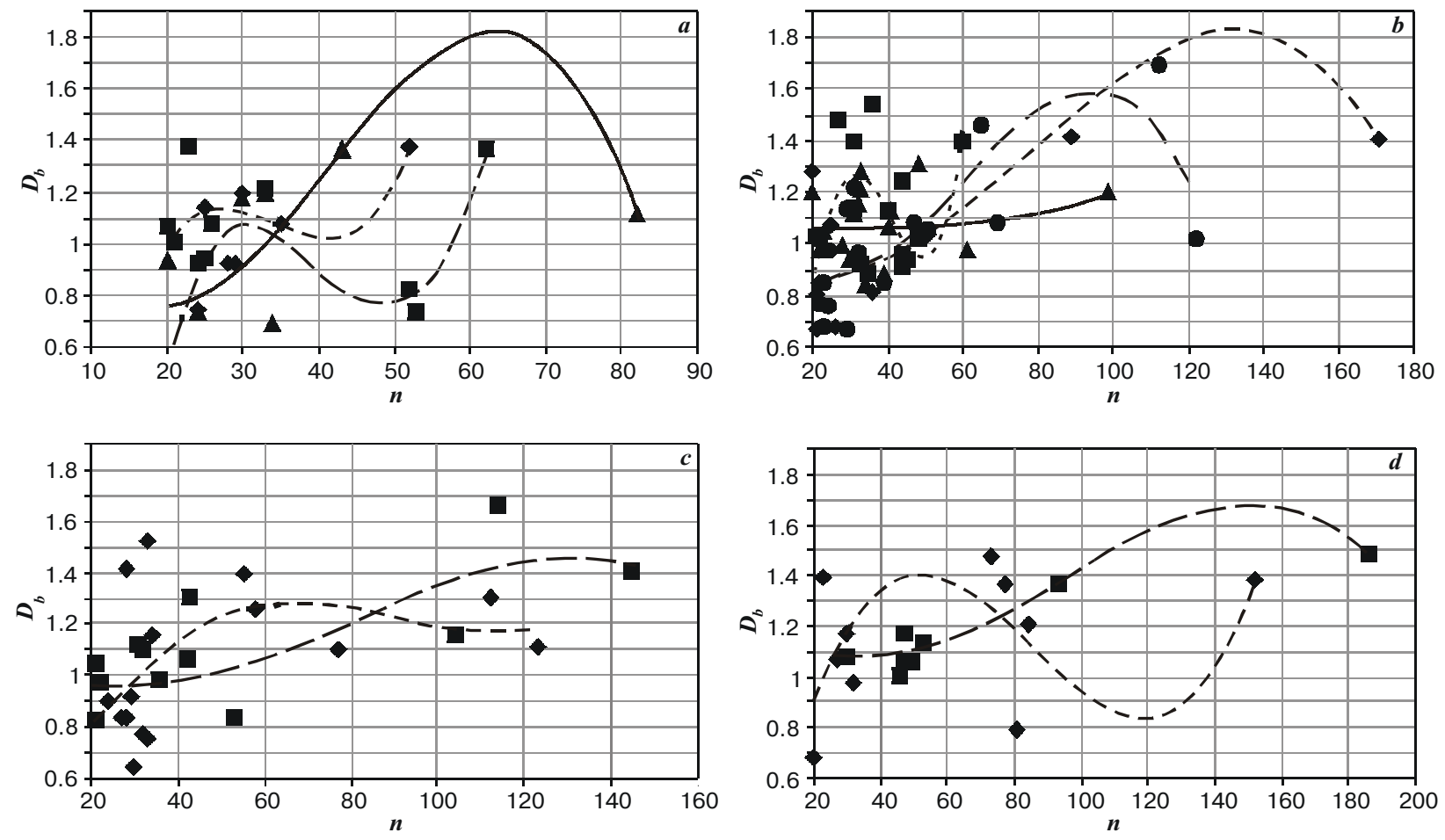

Fig. 4. Fractal dimension for clusters of various sizes $(n)$ obtained at temperatures of $600 \mathrm{~K}, 610 \mathrm{~K}, 620 \mathrm{~K}($ a); $680 \mathrm{~K}$ to $710 \mathrm{~K}(b)$; $730 \mathrm{~K}$, $740 \mathrm{~K}(c)$ and $760 \mathrm{~K}, 770 \mathrm{~K}(d)$ for $N=1000$. 


\section{Pyziak et al.: Topography of Si epitaxial monolayers ...}
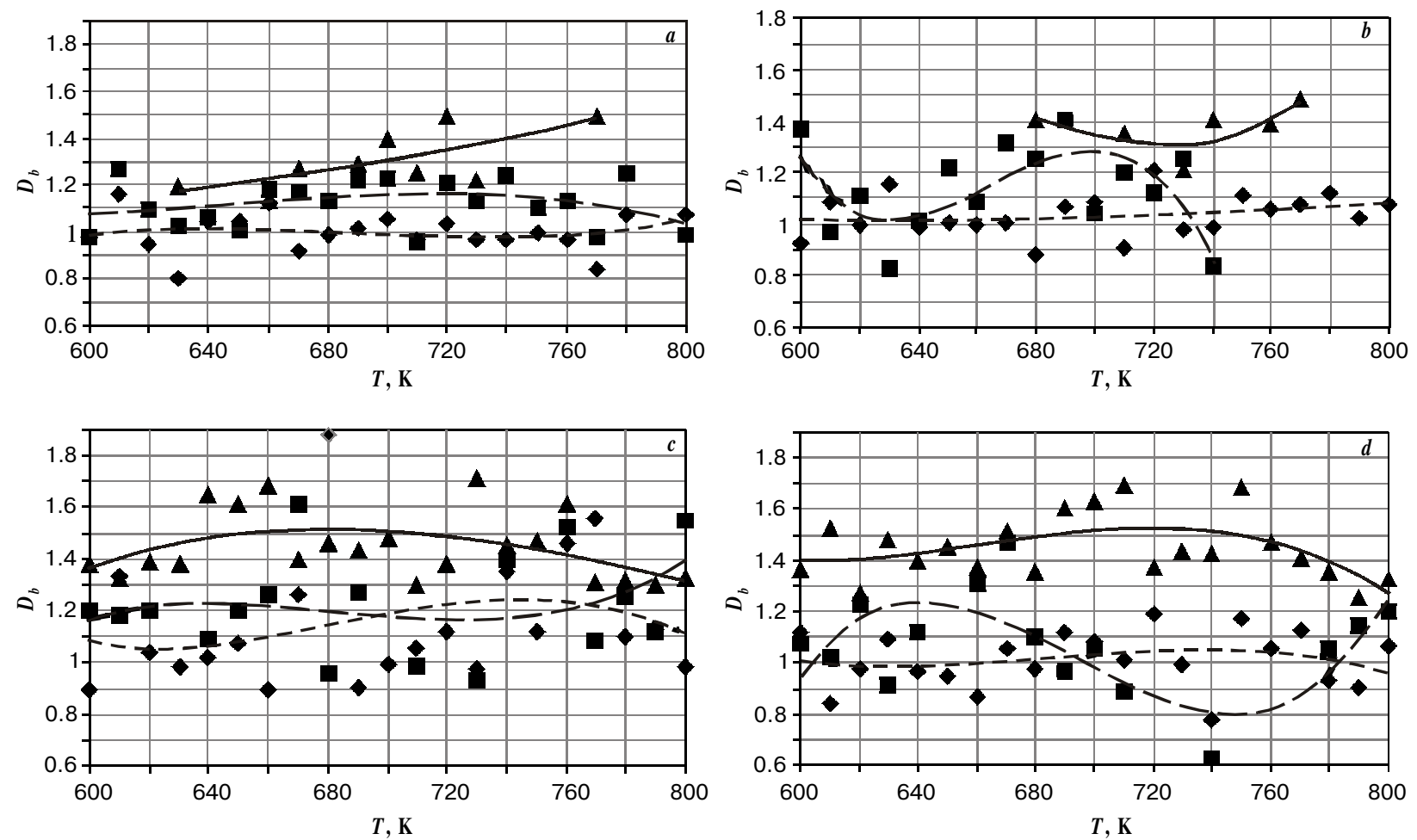

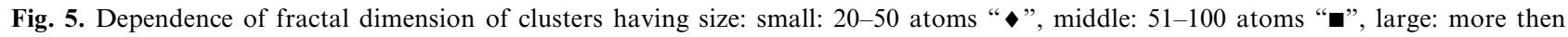
100 atoms " $\Delta$ " on the substrate temperature for $N=1000(a, b)$ and $N=1300(c, d)$. Presented results are for two independent simulations $(a, b)$ and $(c, d)$ obtained for the same parameters

In Fig. 5 we collected averaged fractal dimension (over all clusters which belong to the given size group) depending on the temperature. These results confirm the supposition that larger clusters have greater fractal dimensions. Moreover, in the case of medium sized clusters fractal dimension have a maximum value for temperature 700 to $740 \mathrm{~K}$. For large clusters we find that in the range of temperature from 720 to $770 \mathrm{~K}$ the average fractal dimension is greater and reaches the value of 1.5.

\section{Conclusions}

Monte Carlo simulation of layer growth is a very useful tool for the determination of the growth conditions particularly in the initial stage of crystallisation. A proper adjustment of such conditions is a major task because the structure of the first monolayer strongly influences the further crystallisation: it provides a proper mode of layer growth, an adjustment of the layer lattice constant to the substrate, the number of defects etc. The very important parameter like substrate temperature can by determined easily by this method of simulation as well by fractal analysis of the clusters formed. A notable feature of the approach is the quantitative determination of the shape as well as the geometry of clusters. In the paper we applied the method to the Si layer deposited on (001) Si substrate by pulsed laser ablation. We have determined a "temperature window" being in the range $730-750 \mathrm{~K}$ for the case of the surface density of particles landed: $\sigma_{1}=10^{-14} \mathrm{~cm}^{-2}$ or $\sigma_{2}=1.3 \cdot 10^{-14} \mathrm{~cm}^{-2}$.

For our calculations we assumed that the adatoms were deposited with low kinetic energy. The method presented can by easy adapted to the other methods like e.g. to epitaxy from vapour.

\section{References}

1. D.B. Chrisey, G.K. Hubler (Eds.), Pulsed Laser Deposition of Thin Films, A Wiley-Interscience Publication, New York, 1994.

2. Editors: B. Barren, J.J Dubowski, D.P. Norton, Laser Ablation in Materials Processing, Materials Research Society, Pittsburgh, Pennsylvania, vol.285, 1993.

3. R.T. Tung, J.M. Gibson, J.C. Bean, et al. Appl.Phys. Lett. 40(7), 684-686, 1992,

4. H.A. Blank, H. Rogalla, J. Matter. Res., Vol. 12, No. 11, Nov 1997 ,

5. D.K. Fork, G.B. Anderson, Appl. Phys. Lett. 63(8), 10291031, 1993,

6. H.J. Huang, T. Rabson, Proceedings, ISIF 91 3rd International Symposium on Integrated Ferroelectrics, Colorado Springs, pp. 278-287, 1991.

7. S. Wolf, R. Volz, U. Reichelt, K. Battling, Laser und Optoelektronik 26, 63-67,1994,

8. C.F. Marsden, A.F.A. Haodley, J.D. Wagniere, Proc. ECLAT 90, 543-553, 1990. 


\section{Pyziak et al.: Topography of Si epitaxial monolayers ...}

9. C.M. Cottell, D.B. Chrisey, K.S. Grabowski, in Proceedings, Fall 1991 Meeting of the Materials Research Society, 252 (L.G. Cima, E. Ron, eds.) 3-8, 1992,

10. C.M. Cotell, J.E. Connell, K.C. Dee, R. Bizios, in Transactions of the $19^{\text {th }}$ Annual Meeting of the Society for Biomaterials and $25^{\text {th }}$ International Biomaterials Symposium, Society for Biomaterials, Minneapolis, MN, 103, 1993.

11. C.A.J. Damen, H-J H Smilde, D.H.A. Blank, H. Rogalla, Supercond. Sci. Technol. 11, 437-448, 1998.

12. D. Dijkkamp, T. Venkatesan et al., Appl. Phys. Lett. 51 (8), 619-621, 24 August 1987.

13. X.D. Wu, D. Dijkkamp, S.B. Ogale, A. Inam, E.W. Chase, P.F. Miceli, C.C. Chang, J.M. Tarascon, T. Venkatesan, Appl. Phys. Lett. 51, (11), September1987.

14. G.L. Doll, J.A. Sell, C.A. Taylor II, R. Clarke, Phys. Rev. B43, 6816, 1991,

15. O. Auciello, T. Barnes, S. Chevacharoenkul, A.F. Schreiner, G.E. McGuire, Thin Films 181, 65, 1989,

16. M.G. Norton, P.G. Kotula, C.B. Carter, J. Appl. Phys. 70 , 2871, 1991,

17. A. Frenk, N. Henchoz, W. Kurz, Z. Metallkunde 84, 886892, 1993.

18. C.M. Cottel, Appl. Surf. Phys. 69, 140-148, 1993.

19. C.M. Cottel, D.B. Chrisey, K.S. Grabowski, J.A. Sprague, C.R. Gossett, J. Appl. Biomater. 3, 87-93, 1992a.

20. M. Jelinek, J. Lancok, J. Sonsky et al., Czechoslovak J Phys 48, 5, 577-597, 1998.

1. S.G. Hansen, T.E. Robitaille, Appl. Phys. Lett. 84, 2122, 1988.

22. S.G. Hansen, T.E. Robitaille, J. Appl. Phys. 52, 81, 1988.
23. Dave H.A. Blank, G.J.H.M. Rijndars, G. Koster, H. Rogalla, Apllied Surface Science, 127-129, 633-638, 1998.

24. M. Ohtsuka, Journal of Crystal Growth 205, 112-122, 1999.

25. A. Lo, R.T. Skodje, J. Chem. Phys. 112, 4, 1966-1974, 2000.

26. R.M. Hardeveld, M.J.P. Hopstaken et al., Chem. Phys. Lett. 302, 98-102, 1999.

27. K. Binder, D.W. Heermann, Monte Carlo simulations in statistical Physics. An Introduction, Springer Series in SolidState Sciences, vol. 80 (Springer, Berlin, 1990),

28. D.W. Heermann, Computer simulations methods in theoretical Physics, 2nd ed (Springer, Berlin, 1990).

29. H. Sitter, Thin Solid Films 267, 37-46, 1995.

30. M. Kotrla, Computer Physics Communications 97, 82-100, 1995.

31. M. Kuzma, M. Bester, L. Pyziak, I. Stefaniuk, I. Virt, Applied Surface Science, 168, 132-135, 2000.

32. S.A. Barnett, A. Rockett, Surface Science 198, 133-150, 1988.

33. Z. Zhang, M.G. Lagally, Science, vol.276, April 1997.

34. G.H. Gilmer, H. Huang, C. Roland, Comp. Mater. Sci. 12, 354-380, 1998.

35. S. Kersulis, V. Mitin, Mater. Sci. Eng. B2934, 1995.

36. B.B. Mandelbrot, The fractal geometry of nature (Freeman, New York, 1982).

37. L. Pietronero, E. Tosatti, Eds. Fractal in Physics, International Symposium on Fractals in Physics (6th, 1985,ICTP, Trieste, Italy)

38. H-O. Peitgen, H. Jurgens, D. Saupe, Chaos and fractals. New Frontiers of science, Springer, New York, 1993.

39. R. Kelly, J.E. Rothenberg, Nucl. Instrum. Meth. B7/8, 755,1985 .

40. R. Kelly, J.J. Cuomo, P.A. Leary, J.E. Rothenberg, B.E. Braren, C.F. Aliotta, Nucl. Instrum. Meth. B9, 329,1985. 